\title{
MAGIC studies of the Crab Pulsar and Nebula spectra for energies above $20 \mathrm{GeV}$
}

\author{
Giovanni Ceribella ${ }^{* a b}$, Giacomo D’Amico ${ }^{a}$, Francesco Dazzi ${ }^{c}$, Yuki Iwamura ${ }^{d}$, \\ Marcos López $^{e}$, Jezabel R. Garcia ${ }^{a}$, Takayuki Saito ${ }^{d}$, Thomas Schweizer ${ }^{a}$, \\ Julian Sitarek $^{f}$ for the MAGIC Collaboration ${ }^{\dagger}$ \\ ${ }^{a}$ Max-Plank-Institute for Physics, Munich, Germany \\ ${ }^{b}$ Physics Department, Technical University of Munich, Garching, Germany \\ ${ }^{c}$ INAF Roma, Rome, Italy \\ ${ }^{d}$ Institute for Cosmic Ray Research, University of Tokyo, Japan \\ ${ }^{e}$ Universidad Complutense de Madrid, Madrid, Spain \\ ${ }^{f}$ University of Łódź, Łódź, Poland \\ E-mail: ceribell@mpp.mpg.de, damico@mpp.mpg.de, \\ francesco.dazzi@inaf.it, iwamuraeicrr.u-tokyo.ac.jp, \\ marcos@gae.ucm.es, jezabel@mpp.mpg.de, tschweiz@mpp.mpg.de, \\ tsaito@icrr.u-tokyo.ac.jp, jsitarek@uni.lodz.pl
}

\begin{abstract}
The Crab Nebula is the most studied object in the gamma-ray sky. Yet there are many important unanswered questions on the interrelation between the gamma-ray emission from the Pulsar and the Nebula. We present the results of 112 hours of observations conducted with the MAGIC Telescopes by using the stereoscopic Sum-Trigger-II, a novel trigger system essentially halving the lower energy threshold of the telescopes. In order to process the data, a dedicated analysis aiming for the lowest energies is applied. From these observations, we detect pulsed gammaray emission down to $27 \mathrm{GeV}$, which allows us to perform a detailed spectral study. Due to the sound statistical basis, we perform short-time variability studies of the Pulsar and test possible correlations with the nebular flares detected by Fermi-LAT.
\end{abstract}

36th International Cosmic Ray Conference -ICRC2019-

July 24th - August 1st, 2019

Madison, WI, U.S.A.

\footnotetext{
*Speaker.

†https://magic.mpp.mpg. de/. For collaboration list see PoS(ICRC2019)1177
} 


\section{Introduction}

MAGIC pioneered the Very-High-Energy observations of the Crab Pulsar in 2008 with the discovery, in monoscopic mode, of its pulsed emission above $25 \mathrm{GeV}$ [1]. After the commissioning of the second MAGIC Telescope and in the following years, several major results were achieved by studying the Crab Pulsar, among which the most important ones were the characterisation of the different spectra of the two pulses and the detection of pulsed signal above $1 \mathrm{TeV}$ [2]. With the introduction of the Sum-Trigger-II [3], the MAGIC Telescopes have reached a low energy threshold of around $25 \mathrm{GeV}$ for a Crab-Pulsar-like spectrum. This is similar to the one achieved by the mono Sum-Trigger [1], but profiting from a better trigger efficiency and a more accurate reconstruction of the events, due to the stereoscopy. MAGIC has thus became a very proficient instrument for low-energy sources like pulsars, distant AGNs and GRBs.

\section{Data and Analysis}

We present observations of the Crab Pulsar and Nebula taken with Sum-Trigger-II between 2015 and 2019, which result, after quality cuts, in $112 \mathrm{~h}$ of data. Observations were limited in their zenith-angle to be lower than $Z_{d}=25 \mathrm{deg}$, corresponding to a maximum airmass variation of $10 \%$. We required a minimum atmospheric transmission of $90 \%$, as measured by the MAGIC Lidar, in order to avoid possible bad weather conditions.

The sensitivity of MAGIC at the lowest energies has been further improved by developing a dedicated analysis pipeline with a novel cleaning algorithm, already presented in [4]. Special Monte-Carlo simulations of Extensive Air Showers following the track of the source on the sky were employed to better reconstruct the properties of the primary gamma rays.

\section{Results}

The overall significance of the pulsed emission in a broad range of reconstructed energies between $30 \mathrm{GeV}$ and $200 \mathrm{GeV}$ exceeds $20 \sigma$. Given the square-root growth of the cumulated signal significance with time, this results in an average detection rate of $2.0 \sigma / \sqrt{\mathrm{h}}$. Equivalently, MAGIC can achieve a $5 \sigma$ detection of the VHE pulsed emission in approximately $6 \mathrm{~h}$.

The large statistics at the lower energies enables us to monitor for the first time the VHE flux of the Crab Pulsar over time-scales of few days and probe its stability. This is particularly meaningful, because Fermi/LAT has proven the stability of the Crab Pulsar at energies above $100 \mathrm{MeV}$ [5], but its collection area is too small to provide sufficient statistics to build a light-curve above tens of $\mathrm{GeV}$ on time-scales of few days.

Moreover, the spectrum of both pulses is obtained in the energy range between $27 \mathrm{GeV}$ and $400 \mathrm{GeV}$. Spectra are well modeled by a simple power law and join smoothly with the ones measured by Fermi/LAT over its more than 10 years of operation. The spectrum of the Nebula, detected 
in the range $27 \mathrm{GeV}$ to $15 \mathrm{TeV}$, is also overlapping with the Fermi/LAT one, and gives a powerful cross-calibration tool for the energy scale of the MAGIC Telescopes.

\section{References}

[1] E. Aliu et al., Observation of Pulsed Gamma-rays Above $25 \mathrm{GeV}$ from the Crab Pulsar with MAGIC, Science 322:1221-1224, 2008 [astro-ph/ 0809 . 2998]

[2] S. Ansoldi et al., Teraelectronvolt pulsed emission from the Crab pulsar detected by MAGIC, Astronomy \& Astrophysics, Volume 585, A133, 2016 [astro-ph. HE/ 1510.07048 ]

[3] J. R. Garcia et al., Status of the new Sum-Trigger system for the MAGIC telescopes, in Proceedings of the 33th International Cosmic Ray Conference, astro-ph. IM/1404 . 4219

[4] M. Shayduk, Optimized next-neighbor image cleaning method for Cherenkov Telescopes, in Proceedings of the 33th International Cosmic Ray Conference, astro-ph. IM/1307. 4939

[5] A. A. Abdo et al., Gamma-ray flares from the Crab Nebula, Science 331:739-742, 2011 [astro-ph. HE/1011.3855] 